\title{
A Constitutive Model for Isothermal Pseudoelasticity Coupled with Plasticity
}

\author{
Dongjie Jiang ${ }^{1} \cdot$ Chad M. Landis ${ }^{1}$
}

Published online: 3 October 2016

(C) ASM International 2016

\begin{abstract}
In this paper, a new constitutive model for isothermal pseudoelastic shape memory alloys is presented. The model is based upon a kinematic hardening framework that was previously developed for ferroelastic and ferroelectric switching behavior. The basis of the model includes a transformation surface, an associated flow rule for transformation strain, and kinematic hardening with the back stresses represented by a transformation potential that is dependent upon the transformation strain. In contrast to many models that introduce tension/compression asymmetry by devising transformation surfaces in terms of invariants of the stress tensor, this model achieves this capability by means of expressing the transformation potential from which the back stresses are derived as a weighted mix of two potentials that are, respectively, calibrated to measured tensile and compressive responses. Additionally, in this model, plastic deformation is allowed to occur at high stresses by employing a standard $J_{2}$-based yield surface with isotropic hardening. Finally, to demonstrate the ability of the constitutive model to perform in highly non-proportional loading states, some finite element simulations on crack tip fields are presented.
\end{abstract}

Keywords Pseudoelasticity $\cdot$ Shape memory $\cdot$ SMA

Chad M. Landis

landis@utexas.edu

1 Department of Aerospace Engineering and Engineering Mechanics, The University of Texas at Austin, 210 East 24th Street, C0600, Austin, TX 78712-1221, USA

\section{Introduction}

In relatively high-temperature regimes above the "austenite finish temperature" $A_{\mathrm{f}}$, shape memory alloys (SMAs) have special characteristics of pseudoelasticity, which are caused by diffusionless transformations between two phases austenite and martensite [1]. For an SMA, at such a temperature, the material is initially in the austenitic phase, which has a body-centered cubic (BCC) lattice with a high degree of symmetry. As the material is loaded above some critical level, austenite becomes unstable and stress-induced martensite starts to nucleate. The martensitic phase has a monoclinic lattice structure with a lower degree of symmetry and the transformation from the austenitic BCC lattice to the martensitic one induces a macroscopic deformation that can be described by the Bain matrix [2] From the macroscopic point of view, the amount of strain induced by such martensitic transformations for a SMA is a characteristic material property that can be measured in tests under simple loadings, e.g., uniaxial tension or compression. Upon unloading, when the load decreases below another critical level, the stress-induced martensite cannot exist stably and begins to transform back to austenite. Once the reverse transformation is finished, the microstructure returns to its original state if no dislocation-driven plasticity occurred during the initial loading of the material. Thus, the remaining process of unloading is elastic and finally the deformation would recover completely.

One important feature of SMA pseudoelastic behavior is the significant asymmetry between tension and compression. Gall et al. [3] reported distinct tensile and compressive responses of precipitated NiTi crystals, which are also dependent on crystallographic orientation with respect to the loading direction. Bechle and Kyriakides [4] conducted carefully designed isothermal tensile and compressive tests 
on polycrystalline NiTi tubes, which show that the stressinduced martensitic transformation occurs at a much higher stress level but with only about half amount of transformation strain (the inelastic strain induced by transformation that can be recovered upon unloading) under compression compared to those achieved under tension. In addition, the deformation in the specimen under tension is highly inhomogeneous, exhibiting propagating Luders-like bands of localized transformed zones. Reedlunn et al. [5] disclose similar experimental observations.

For the purpose of facilitating theoretical and numerical modeling studies of the behaviors of SMA structures, numerous studies have been published on the phenomenological constitutive modeling of SMAs. (The number of publications on SMA constitutive modeling is too large for us to cite all studies on this topic. Hence, we only cite those that are directly related to this paper.) Many of the phenomenological constitutive models developed to date are plasticity-based [6, 7]. Boyd and Lagoudas [8] initially proposed a model for SMAs with the free energy density being a sum of free energy densities of the two phases weighted by the martensite volume fraction in addition to a contribution from the mix of the two phases. A series of subsequent extensions of this model were published, among which Qidwai and Lagoudas [9] introduced several transformation functions expressed in terms of the three invariants of the stress tensor in order to enable tension/compression asymmetry and volumetric transformation strain. Auricchio and Petrini [10] developed a threedimensional model for stress-temperature-induced solidphase transformation with the transformation strain as the internal variable, which includes a Prager-Lode-type limit surface to induce different critical stresses under tension and compression. Using a similar Helmholtz free energy, Arghavani et al. [11] presented a model in which the amount and orientation of the transformation strain evolve separately. Both models proposed by Arghavani et al. [12] and Panico and Brinson [13] take the stress-induced martensite fraction and the temperature-induced martensite fraction as separate internal variables and describe martensite reorientation separately from austenite to martensite transformation. In addition to these yield surface plasticity-based approaches, the micro-plane modeling approach has also been applied to SMAs. By modeling the effects of transformation-induced deformation on several planes of different orientations, the micro-plane model proposed by Brocca et al. [14] is able to predict the threedimensional responses of SMAs. Karamooz Ravari et al. [15] extended the micro-plane modeling approach to incorporate the tension/compression asymmetry by adopting an equivalent stress based on the second and third invariants of the deviatoric stress tensor.
In this paper, a new constitutive model for isothermal pseudoelastic SMAs is presented. The framework of the model inherits the one previously developed for ferroelastic and ferroelectric switching behavior, see Landis [16-18]. Rather than distinguishing between the austenite to martensite transformation and the reorientation of martensite, the model takes the transformation strain as the sole internal variable. The basis of the model includes a transformation surface, an associated flow rule for transformation strain, and kinematic hardening with back stress derived from a transformation potential that is dependent upon the transformation strain. In contrast to most models that introduce tension/compression asymmetry by devising transformation surfaces in terms of invariants of the stress tensor, this model achieves this capability by means of expressing the transformation potential as a weighted mix of two potentials that are, respectively, calibrated to measured tensile and compressive responses. Additionally, like other metallic materials, plasticity also occurs in SMAs once dislocation slip is activated, see Sehitoglu et al. [19], Ezaz et al. [20], Hartl and Lagoudas [21]. Thus, in this model, plastic deformation is allowed to occur at high stresses by employing a standard $J_{2}$-based yield surface with isotropic hardening. To demonstrate of the ability of the constitutive model to integrate highly non-proportional loading states, finite element simulations on stationary and steadily propagating cracks are conducted and presented.

The organization of this paper is as follows: "Constitutive Model for Pseudoelastic Shape Memory Alloys" section presents the formulation of the proposed constitutive model for isothermal pseudoelastic behaviors of SMAs. "Results for Boundary Value Problems" section demonstrates the results of the finite element simulations on the crack tip field for a stationary crack and that for a steadily propagating crack, in which the constitutive model is applied. Finally, "Discussion" section summarizes the constitutive model developed and the example boundary value problems on crack tip fields that are analyzed with this model.

\section{Constitutive Model for Pseudoelastic Shape Memory Alloys}

\section{Framework}

Let $\sigma_{i j}$ and $s_{i j}$ be the stress tensor and its deviatoric components. The strain tensor is decomposed into elastic, transformation, and plastic strains, i.e., $\varepsilon_{i j}=\varepsilon_{i j}^{e}+\varepsilon_{i j}^{t}+\varepsilon_{i j}^{p}$. The following Helmholtz free energy per unit volume is introduced:

$\psi=\frac{1}{2} c_{i j k l}\left(\varepsilon_{i j}-\varepsilon_{i j}^{t}-\varepsilon_{i j}^{p}\right)\left(\varepsilon_{k l}-\varepsilon_{k l}^{t}-\varepsilon_{k l}^{p}\right)+\psi^{t}\left(\varepsilon_{i j}^{t}\right)$, 
in which the first term is the elastic strain energy stored per unit volume and the second term represents the component of the free energy attributed to phase transformation. $c_{i j k l}$ are the components of the linear elastic stiffness tensor, which is possibly dependent on the transformation strain, i.e., $c_{i j k l}=c_{i j k l}\left(\varepsilon_{m n}^{t}\right)$.

The isothermal form of the second law of thermodynamics requires that the dissipation rate for any possible process must be non-negative, i.e.,

$\dot{\Delta}=\sigma_{i j} \dot{\varepsilon}_{i j}-\dot{\psi} \geq 0$.

Together with the following definitions,

$\bar{\sigma}_{i j}=\frac{1}{2} \frac{\partial s_{p q r s}}{\partial \varepsilon_{i j}^{t}} \sigma_{p q} \sigma_{r s}$

$\sigma_{i j}=\frac{\partial \psi}{\partial \varepsilon_{i j}}=c_{i j k l}\left(\varepsilon_{k l}-\varepsilon_{k l}^{t}-\varepsilon_{k l}^{p}\right)$,

$\sigma_{i j}^{B}=\frac{\partial \psi^{t}}{\partial \varepsilon_{i j}^{t}}$

$\hat{\sigma}_{i j}=\sigma_{i j}-\sigma_{i j}^{B}+\bar{\sigma}_{i j}$

the second law expressed in (2) may be re-organized as

$\dot{\Delta}=\hat{\sigma}_{i j} \dot{\varepsilon}_{i j}^{t}+\sigma_{i j} \dot{\varepsilon}_{i j}^{p} \geq 0$.

Note that the $s_{i j k l}$ are the components of the elastic compliance tensor, which is the inverse of the elastic stiffness tensor, i.e., $s_{i j k l}=\left(c_{i j k l}\right)^{-1}$.

It is assumed that elastic deformation is enclosed by both a transformation surface $\Phi^{t}\left(\hat{\sigma}_{i j}, \varepsilon_{i j}^{t}\right)=0$ and a yield surface $\Phi^{p}\left(\sigma_{i j}, \bar{\varepsilon}^{p}\right)=0$, and transformation (plastic yielding) may occur if $\hat{\sigma}_{i j}\left(\sigma_{i j}\right)$ lies on the transformation (yield) surface. The variable $\bar{\varepsilon}^{p}$ is the accumulated plastic strain defined as,

$\overline{\bar{\varepsilon}}^{p}=\int_{0}^{t}\left(\frac{2}{3} \dot{\varepsilon}_{i j}^{p} \dot{\varepsilon}_{i j}^{p}\right)^{1 / 2} d t$.

Stress states outside either the transformation or the yield surface are forbidden. It is assumed that both transformation and plastic straining abide by the maximum dissipation postulate, and hence both surfaces must be convex and the transformation and plastic strain increments follow associative flow rules,

$\dot{\varepsilon}_{i j}^{t}=\lambda^{t} \frac{\partial \Phi^{t}}{\partial \hat{\sigma}_{i j}}$,

$\dot{\varepsilon}_{i j}^{p}=\lambda^{p} \frac{\partial \Phi^{p}}{\partial \sigma_{i j}}$.

Equations (6) and (7) indicate that the transformation strain increment and plastic strain increment are normal to corresponding surfaces. Additionally, $\lambda^{t}$ and $\lambda^{p}$ are nonnegative multipliers that are determined from consistency conditions. Note that along with the maximum dissipation postulate which implies convexity of the surface and normality for the flow rules, (4) is then satisfied as long as $\Phi^{t}\left(\hat{\sigma}_{i j}=0, \varepsilon_{i j}^{t}\right) \leq 0$ and $\Phi^{p}\left(\sigma_{i j}=0, \bar{\varepsilon}^{p}\right) \leq 0$. The loading and unloading conditions can also be recast as the KuhnTucker conditions,

$\lambda^{t} \geq 0, \quad \Phi^{t} \leq 0, \quad \lambda^{t} \Phi^{t}=0$

$\lambda^{p} \geq 0, \quad \Phi^{p} \leq 0, \quad \lambda^{p} \Phi^{p}=0$.

Note that for this specific variant of the theoretical framework, we have not accounted for the possibility of transformation-induced plasticity. The physics of transformation-induced plasticity can be included within this framework in a manner similar to that proposed by Lagoudas and Entchev [22], wherein a contribution of the plastic strain increment is linked to the transformation strain increment. The details of such modifications are beyond the scope of this work, and as such, the present model is most applicable to materials where transformation-induced plasticity is negligible (e.g., as in [4, 23, 24]).

To derive the continuum tangent behavior for this model, we define,

$U_{i j k l}=\frac{1}{2} \frac{\partial^{2} s_{p q r s}}{\partial \varepsilon_{i j}^{t} \partial \varepsilon_{k l}^{t}} \sigma_{p q} \sigma_{r s}$,

$H_{i j k l}=\frac{\partial^{2} \psi^{t}}{\partial \varepsilon_{i j}^{t} \partial \varepsilon_{k l}^{t}}$,

$\tilde{\varepsilon}_{i j}=\frac{\partial \Phi^{t}}{\partial \hat{\sigma}_{i j}}+\frac{\partial \Phi^{t}}{\partial \hat{\sigma}_{k l}} \frac{\partial s_{m n i j}}{\partial \varepsilon_{k l}^{t}} \sigma_{m n}$,

in which case the consistency conditions, $\dot{\Phi}^{t}=0$ and $\dot{\Phi}^{p}=0$, lead to the following equations:

$\left(\begin{array}{ll}A^{t t} & A^{t p} \\ A^{p t} & A^{p p}\end{array}\right)\left[\begin{array}{l}\lambda^{t} \\ \lambda^{p}\end{array}\right]=\left[\begin{array}{l}b^{t} \\ b^{p}\end{array}\right]$

where

$A^{t t}=\tilde{\varepsilon}_{i j} c_{i j k l} \tilde{\varepsilon}_{k l}+\frac{\partial \Phi^{t}}{\partial \hat{\sigma}_{i j}}\left(H_{i j k l}-U_{i j k l}\right) \frac{\partial \Phi^{t}}{\partial \hat{\sigma}_{k l}}-\frac{\partial \Phi^{t}}{\partial \varepsilon_{i j}^{t}} \frac{\partial \Phi^{t}}{\partial \varepsilon_{i j}^{t}}$,

$A^{t p}=A^{p t}=\tilde{\varepsilon}_{i j} c_{i j k l} \frac{\partial \Phi^{p}}{\partial \sigma_{k l}}$,

$A^{p p}=\frac{\partial \Phi^{p}}{\partial \sigma_{i j}} c_{i j k l} \frac{\partial \Phi^{p}}{\partial \sigma_{k l}}-\frac{\partial \Phi^{p}}{\partial \bar{\varepsilon}^{p}} \sqrt{\frac{2}{3} \frac{\partial \Phi^{p}}{\partial \sigma_{i j}} \frac{\partial \Phi^{p}}{\partial \sigma_{i j}}}$,

$b^{t}=\tilde{\varepsilon}_{i j} c_{i j k l} \dot{\varepsilon}_{k l}$, and

$b^{p}=\frac{\partial \Phi^{p}}{\partial \sigma_{i j}} c_{i j k l} \dot{\varepsilon}_{k l}$ 
The multipliers $\lambda^{t}$ and $\lambda^{p}$ are solved from (12), given the variables at the current state and the strain increment $\dot{\varepsilon}_{i j}$. Note that if both $\Phi^{t}<0$ and $\Phi^{p}<0$ then both $\lambda^{t}=0$ and $\lambda^{p}=0$ and exhibit elastic behavior. If $\Phi^{t}=0$ and $\Phi^{p}<0$ then $\lambda^{p}=0$ and $\lambda^{t}$ is solved from (12) using $\lambda^{p}=0$. The solution for the multipliers is then used in (6) and (7), which are in turn applied within the rate form of (3b) to obtain the continuum stress increment versus strain increment behavior. The solution to Eq. (12) can be written in the following form,

$$
\begin{gathered}
\lambda^{t}=L_{m n}^{t} \dot{\varepsilon}_{m n} \\
\lambda^{p}=L_{m n}^{p} \dot{\varepsilon}_{m n} .
\end{gathered}
$$

Then the incremental stress-strain relationships are,

$\dot{\sigma}_{i j}=\left(c_{i j k l}-c_{i j m n} \frac{\partial \Phi^{t}}{\partial \hat{\sigma}_{m n}} L_{k l}^{t}-c_{i j m n} \frac{\partial \Phi^{p}}{\partial \sigma_{m n}} L_{k l}^{p}\right) \dot{\varepsilon}_{k l}$.

The terms within the parentheses are the components of the continuum tangent modulus.

\section{Model Fitting to a Material}

The material properties that need to be specified for the constitutive model include the dependence of the elastic properties on the transformation strain, the transformation surface $\Phi^{t}$, the yield surface $\Phi^{p}$, and the back stress potential $\psi^{t}$. For the sake of simplicity, we assume that the elastic properties are independent of the transformation strain and represented by a constant Young's modulus $E$ and Poisson's ratio $v$. As a result, $\bar{\sigma}_{i j}$ and $U_{i j k l}$ vanish. The following $J_{2}$-type transformation surface $\Phi^{t}$ and yield surface $\Phi^{p}$ are adopted,

$\Phi^{t}=\frac{3}{2} \hat{s}_{i j} \hat{s}_{i j}-\sigma_{0}^{2}$

$\Phi^{p}=\frac{3}{2} s_{i j} s_{i j}-\sigma_{y}^{2}$,

where $\sigma_{0}$ and $\sigma_{y}$ represent the sizes of the two surfaces. In contrast to using separate forward and backward transformation surfaces as is done in many other approaches, in this theory, the evolution of the transformation strain is based upon a kinematic hardening description using a single transformation surface. For this approach, the width of the hysteresis loop for uniaxial loading is simply the diameter of the transformation surface, hence the radius of the transformation surface $\sigma_{0}$ in Eq. (20) must be half of the size of the pseudoelastic hysteresis and can in general depend upon the transformation strain state within this theory. However, here $\sigma_{0}$ is assumed to be a constant based on the experimental observations measured in Jiang et al.
[23]. The yield strength $\sigma_{y}$ is explicitly specified as a function of the cumulative plastic strain $\bar{\varepsilon}^{p}$,

$\sigma_{y}\left(\bar{\varepsilon}^{p}\right)=\sigma_{y}^{0}\left[1+\frac{2}{\pi} h_{1}^{p} \tan ^{-1}\left(b^{p} \bar{\varepsilon}^{p}\right)\right]$.

This phenomenological form for the yield strength is chosen to fit the experimental observations obtained in Jiang et al. [23]. Other forms for the yield strength can of course be used.

Recall that the back stress is related to the transformation strain through a potential $\psi^{t}$, as shown in Eq. (3c). The tension/compression asymmetry in the stress-strain behavior and differences between the tensile and compressive saturation strains are both captured with the appropriate design of this potential function. Thus, we propose the following representation of this potential,

$\psi^{t}=\xi \psi_{c}^{t}(\bar{\varepsilon})+(1-\xi) \psi_{t}^{t}(\bar{\varepsilon})$,

where $\psi_{c}^{t}$ and $\psi_{t}^{t}$ are potentials that are calibrated to the measured uniaxial compression and tension stress-transformation strain responses, $\bar{\varepsilon}$ is a strain-like variable that governs the strain asymmetry, and the weighting between the two potentials in the mixture is specified by $\xi$. The following definition of $\bar{\varepsilon}$ is adopted,

$\bar{\varepsilon}=J_{2}^{e} f\left(J_{r}\right), \quad J_{r}=J_{3}^{e} / J_{2}^{e}$,

with

$f=\cos \left\{\arccos \left[1-a\left(J_{r}^{3}+1\right)\right] / 3\right\}$,

where the following definitions of the deviatoric strain invariants are used,

$$
\begin{aligned}
& J_{2}^{e}=\left(2 e_{i j}^{t} e_{i j}^{t} / 3\right)^{1 / 2}, \quad J_{3}^{e}=\left(4 e_{i j}^{t} e_{j k}^{t} e_{k i}^{t} / 3\right)^{1 / 3}, \\
& \text { and } e_{i j}^{t}=\varepsilon_{i j}^{t}-\varepsilon_{k k}^{t} \delta_{i j} / 3 .
\end{aligned}
$$

The function $f$, originally proposed by Sedlak et al. [25], scales the uniaxial compressive transformation strain to its tensile counterpart. In several prior works, the introduction of tension/compression asymmetry is accomplished via the construction of the shape of the transformation surface e.g., [9, 10, 15, 26-29]. In contrast, this approach implements a spherically shaped transformation surface with anisotropic kinematic hardening that accounts for the differing behaviors in tension and compression as the transformation strain evolves.

The weight function $\xi$, chosen to be a function of the ratio of the deviatoric strain invariants $J_{r}$, facilitates the generalization of the uniaxial tensile and compressive responses to arbitrary strain states. $J_{r}=1$ represents the uniaxial extension and $J_{r}=-1$ represents the uniaxial contraction. Thus, $\xi\left(J_{r}\right)$ with 
$\xi(-1)=1$ and $\xi(1)=0$,

interpolates between the tensile and compressive responses. In addition, to ensure continuity of the hardening moduli, the derivatives of $\xi\left(J_{r}\right)$ must satisfy $\xi_{0}^{I}=\xi_{0}^{I I}=\xi_{0}^{I V}=\xi_{0}^{V}=0$, where $\xi_{0}^{n}$ represents the $n$th derivative of $\xi$ with respect to $J_{r}$ at $J_{r}=0$. Similarly, the derivatives of $f\left(J_{r}\right)$ must also satisfy the same conditions, see [17].

According to (3c) and (23), the back stress becomes,

$\sigma_{i j}^{B}=\xi \frac{d \psi_{c}^{t}}{d \bar{\varepsilon}} \frac{\partial \bar{\varepsilon}}{\partial \varepsilon_{i j}^{t}}+(1-\xi) \frac{d \psi_{t}^{t}}{d \bar{\varepsilon}} \frac{\partial \bar{\varepsilon}}{\partial \varepsilon_{i j}^{t}}+\xi^{\prime}\left(J_{r}\right)\left(\psi_{c}^{t}-\psi_{t}^{t}\right) \frac{\partial J_{r}}{\partial \varepsilon_{i j}^{t}}$.

Mathematical derivations indicate that under uniaxial stress states, Eq. (28) becomes

$\sigma_{11 c}^{B}=\frac{d \psi_{c}^{t}}{d \bar{\varepsilon}} \frac{\partial \bar{\varepsilon}}{\partial \varepsilon_{11}^{t}}$ and $\sigma_{11 t}^{B}=\frac{d \psi_{t}^{t}}{d \bar{\varepsilon}} \frac{\partial \bar{\varepsilon}}{\partial \varepsilon_{11}^{t}}$

for compression and tension, respectively. If the plastic yield limit has not been reached, the equation of the transformation surface during loading implies

$\left|\sigma_{11 c}\right|-f(-1) \frac{d \psi_{c}^{t}}{d \bar{\varepsilon}}=\sigma_{0}$ and $\sigma_{11 t}-f(1) \frac{d \psi_{t}^{t}}{d \bar{\varepsilon}}=\sigma_{0}$

where again $\sigma_{0}$ is the size of the transformation surface. Equation (30) clearly demonstrates the capability of this constitutive model to produce arbitrarily different back stress-transformation strain relations under compression and tension.

The function $\xi$ extends the calibrated model to general strain states and ultimately should be calibrated to experimental results. The following expression has been adopted for the calculations presented here which satisfies condition (14) and the requirements on its derivatives at $J_{r}=0$,

$\xi\left(J_{r}\right)=\frac{f\left(J_{r}\right)-f(1)}{f(-1)-f(1)}$.

\section{Parameters of the Model}

The two back stress potentials in (11) are assigned the following forms,

$$
\begin{aligned}
\psi_{c}^{t}(\bar{\varepsilon}) & =\frac{h_{0 c}}{2} \bar{\varepsilon}^{2}+\left(h_{1 c}-h_{0 c}\right)\left\{\frac{\bar{\varepsilon}^{2}}{2}-\frac{1}{b_{c}}\left[\bar{\varepsilon}-\frac{1}{b_{c}}\left(1-e^{-b_{c} \bar{\varepsilon}}\right)\right]\right\} \\
& +\left(h_{2 c}-h_{1 c}\right)\left\{\begin{array}{cr}
0 & 0 \leq \bar{\varepsilon} \leq \varepsilon_{1} \\
\left(\varepsilon_{c}-\varepsilon_{1}\right)^{2}\left(\xi^{5} / 2-\xi^{6} / 2+\xi^{7} / 7\right) & \varepsilon_{1} \leq \bar{\varepsilon} \leq \varepsilon_{c} \\
\left(\varepsilon_{c}-\varepsilon_{1}\right)^{2} / 7+\left(\varepsilon_{c}-\varepsilon_{1}\right)\left(\bar{\varepsilon}-\varepsilon_{c}\right)+\left(\bar{\varepsilon}-\varepsilon_{c}\right)^{2} / 2 & \varepsilon_{c} \leq \bar{\varepsilon}
\end{array}\right.
\end{aligned}
$$

and

$$
\begin{array}{rl}
\psi_{t}^{t}(\overline{\bar{\varepsilon}}) & =\frac{h_{0 t}}{2} \bar{\varepsilon}^{2}+\left(h_{1 t}-h_{0 t}\right)\left\{\frac{\bar{\varepsilon}^{2}}{2}-\frac{1}{b_{t}}\left[\bar{\varepsilon}-\frac{1}{b_{t}}\left(1-e^{-b_{t} \overline{\bar{\varepsilon}}}\right)\right]\right\} \\
0 & 0 \leq \bar{\varepsilon} \leq \varepsilon_{1}
\end{array}
$$

where $\xi=\left(\bar{\varepsilon}-\varepsilon_{1}\right) /\left(\varepsilon_{c}-\varepsilon_{1}\right)$.

The parameters in (22), (32), and (33) are listed in Table 1. Together with $a=0.99225$ in (25), the choice of $\psi_{c}^{t}=\psi_{t}^{t}$, as shown by the parameters listed in Table 1, produces the asymmetric tensile and compressive responses demonstrated in Fig. 1 (with plasticity prevented by enforcing $\varepsilon_{i j}^{p}=0$ ) and Fig. 2 (if plastic yielding is allowed to occur). This choice of $\psi_{c}^{t}=\psi_{t}^{t}$ does in fact capture the experimentally observed tension/compression asymmetry reported in Jiang et al. [23, 24]. Note that for this material, the transformation in compression occurs at a higher stress level than the transformation in tension. This material model can also capture the opposite case. For example, if within the function $f$ in Eq. (25) the term $J_{r}^{3} \rightarrow-J_{r}^{3}$, then the tensile and compressive stress-strain behaviors in Fig. 1 would be interchanged.

Again, note that the radius of the transformation surface is taken to be half of the stress hysteresis, and is therefore smaller than the apparent transformation stress in either tension or compression. The agreement of the model to experimentally observed stress-strain behaviors is then obtained from the transformation potential. In a fashion akin to the determination of experimentally measured "apparent" transformation surfaces, simulations of the modeled apparent transformation surfaces can also be obtained. Figure 3 illustrates the transformation surfaces produced by the constitutive model for several values of the transformation work, $\Delta W^{t}=\int \sigma_{i j} d \varepsilon_{i j}^{t}$, in the biaxial stress $\left(\sigma_{11}-\sigma_{22}\right)$ space. Each point on any of these surfaces is obtained from a proportional load path in stress space, and the stress state at the associated level of transformation work is recorded. The transformation surfaces from the model have a shape that is similar to those

Table 1 The parameter values for the material model calibration used to fit the experimental measurements in [23]

\begin{tabular}{llll}
\hline Parameter (unit) & Value & Parameter (unit) & Value \\
\hline$E(\mathrm{GPa})$ & 68.3 & $h_{2 c}, h_{2 t}(\mathrm{GPa})$ & 603.3 \\
$v$ & 0.30 & $\varepsilon_{1}(\%)$ & 3.1 \\
$\sigma_{0}(\mathrm{MPa})$ & 120.7 & $\varepsilon_{c}(\%)$ & 3.3 \\
$b_{c}, b_{t}$ & 2500 & $\sigma_{y}^{0}(\mathrm{GPa})$ & 1.24 \\
$h_{0 c}, h_{0 t}(\mathrm{GPa})$ & 1508 & $h_{1}^{p}$ & 0.446 \\
$h_{1 c}, h_{1 t}(\mathrm{GPa})$ & 2.772 & $b^{p}$ & 1650 \\
\hline
\end{tabular}




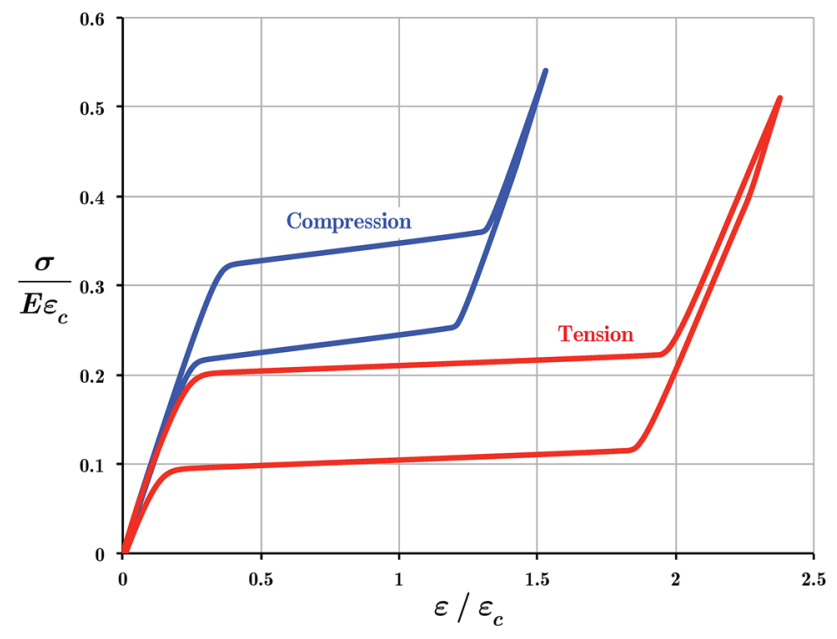

Fig. 1 Uniaxial tensile and compressive responses with pseudoelastic transformation only. Table 1 lists the fitted parameter values to experimental measurements for material model calibration by Jiang et al. $[23,24]$

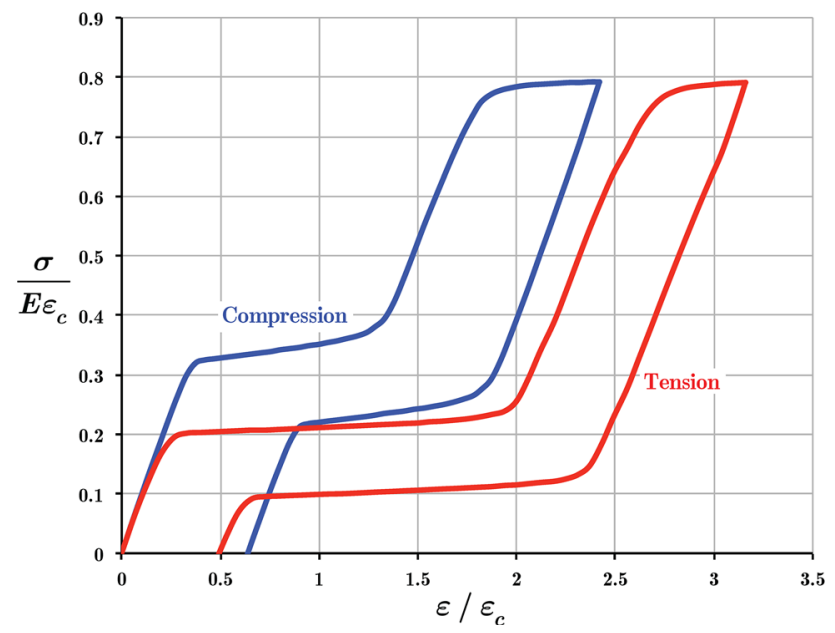

Fig. 2 Uniaxial tensile and compressive responses with both pseudoelastic transformation and plasticity for a material described by the parameter values listed in Table 1

reported in experimental studies (see Lexcellent et al. [26]) and micromechanical simulations, Aleong et al. [30].

\section{Results for Boundary Value Problems}

This constitutive model has been applied within the commercial finite element code ABAQUS ${ }^{\circledR}$ as a UMAT in order to analyze the recoverable buckling of NiTi tubes, Jiang et al. $[23,24]$. These works have demonstrated that this model's ability to describe the asymmetries in the tensile and compressive strains, the tensile and compressive stresses, plasticity at high stresses, and the integration of highly nonproportional stress states are all necessary for capturing the

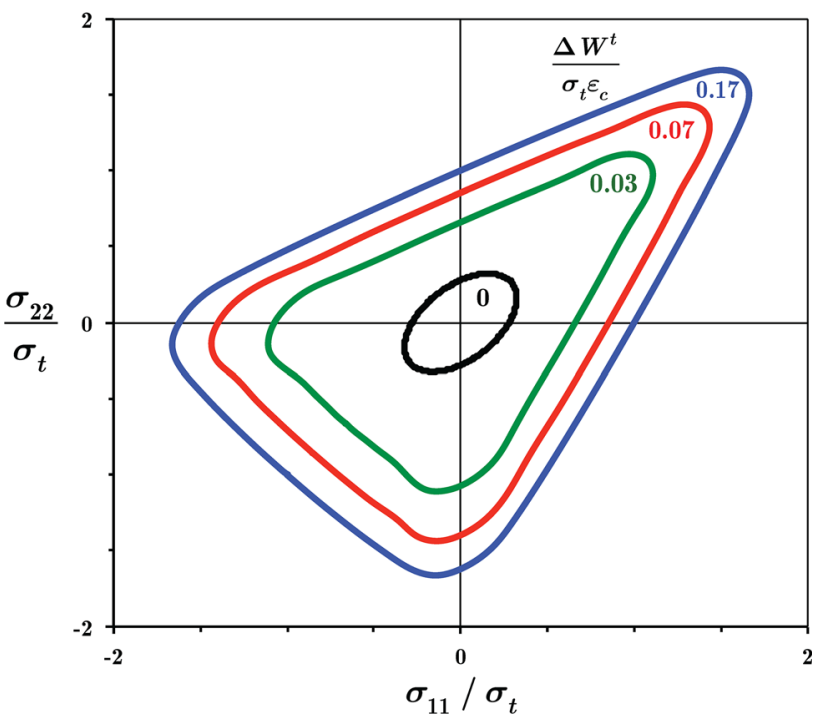

Fig. 3 Transformation surfaces for different values of the transformation work in biaxial stress space

complex structural response of buckling tubes. In this paper, we illustrate some of these behaviors of the model for capturing the fields near stationary and steadily growing crack tips. For this purpose, the same backward Euler integration routine that is required for the ABAQUS UMAT was ported to the finite element codes used to analyze semi-infinite stationary and steadily growing cracks. These results are described in detail in the following subsections.

\section{Stationary Cracks}

In this sub-section, a stationary, semi-infinite crack in a pseudoelastic material is analyzed in a manner similar to Baxevanis et al. [31]. This problem is characterized by a region of transformation around the crack tip, which may also encapsulate a region of plasticity within it. Outside the austenite to martensite transformation region, the material is linear elastic and far from the transformation zone the elastic stress field is described by the mode I $K$-field. The far-field stresses for the $K$-field with polar coordinates centered on the crack tip are given as,

$\left\{\begin{array}{l}\sigma_{x x} \\ \sigma_{y y} \\ \sigma_{x y}\end{array}\right\}=\frac{K_{\mathrm{I}}}{\sqrt{2 \pi r}} \cos \frac{\theta}{2}\left\{\begin{array}{l}1-\sin \frac{\theta}{2} \sin \frac{3 \theta}{2} \\ 1+\sin \frac{\theta}{2} \sin \frac{3 \theta}{2} \\ \sin \frac{\theta}{2} \cos \frac{3 \theta}{2}\end{array}\right\}$.

Instead of constructing a finite element model of a large domain to approximate the semi-infinite crack geometry, a Dirichlet to Neumann map, Carka et al. [32], is applied to the outer boundary of a small circular domain that simply must surround the austenite to martensite transformation zone. This technique allows for a highly concentrated mesh around 

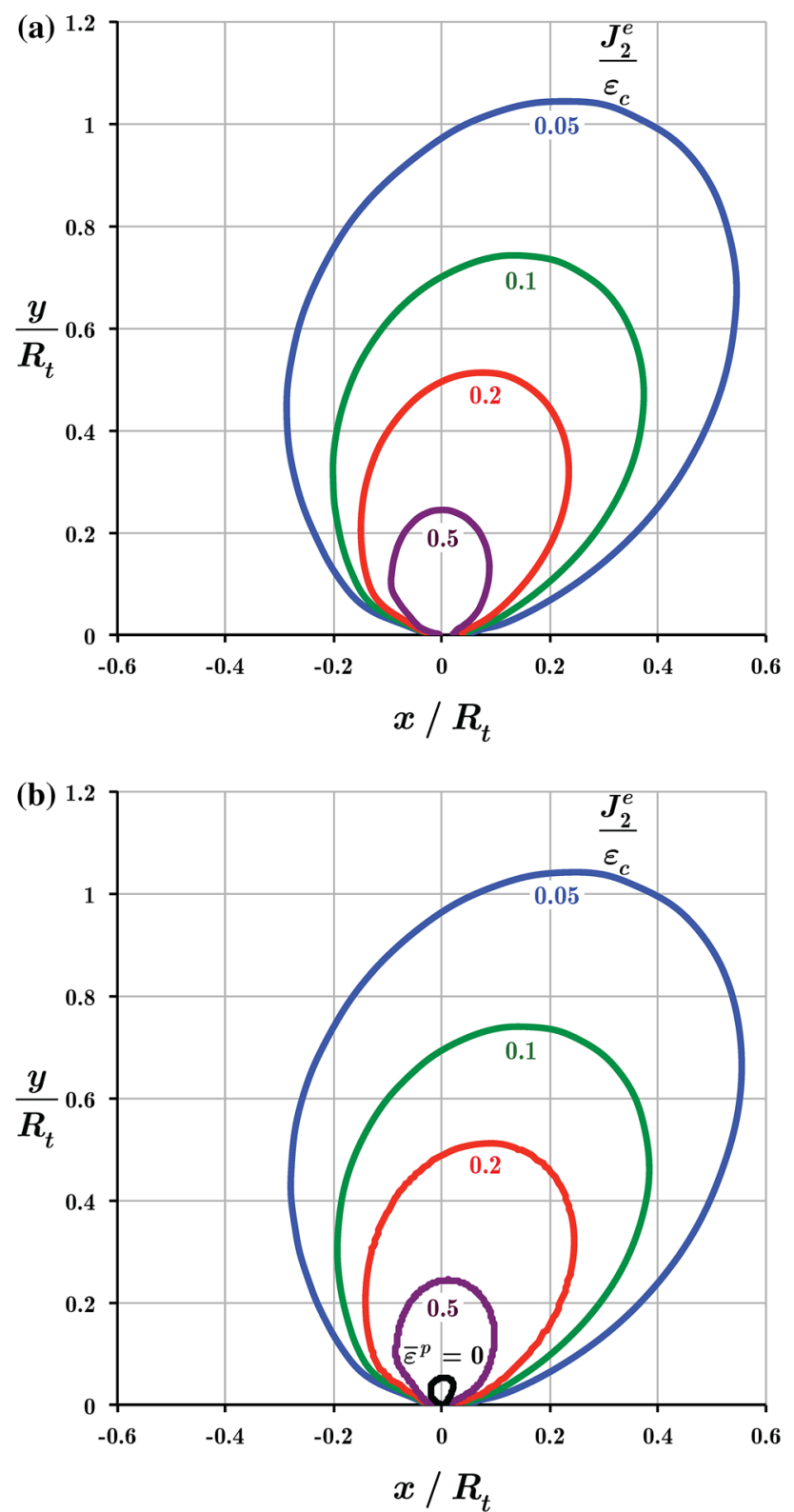

Fig. 4 Solutions around a semi-infinite crack in a pseudoelastic material loaded: a Contours of equivalent transformation strain in the vicinity of a crack tip when the material has pseudoelastic transformation but no plastic deformation. b Contours of equivalent transformation strain and the plastic zone in the vicinity of a crack tip when the material has both pseudoelastic transformation and plastic deformation

the crack tip such that all of the numerical effort can be expended in and around the non-linear transformation zone.

For the presentation of the results, the size of the transformation zone can be approximated by the formula,

$R_{t}=\frac{1}{3 \pi}\left(\frac{K_{I}}{\sigma_{t}}\right)^{2}$,

where $\sigma_{t}=0.2 E \varepsilon_{c}$ is the approximate transformation stress in tension taken from Fig. 1. Figure 4 shows several

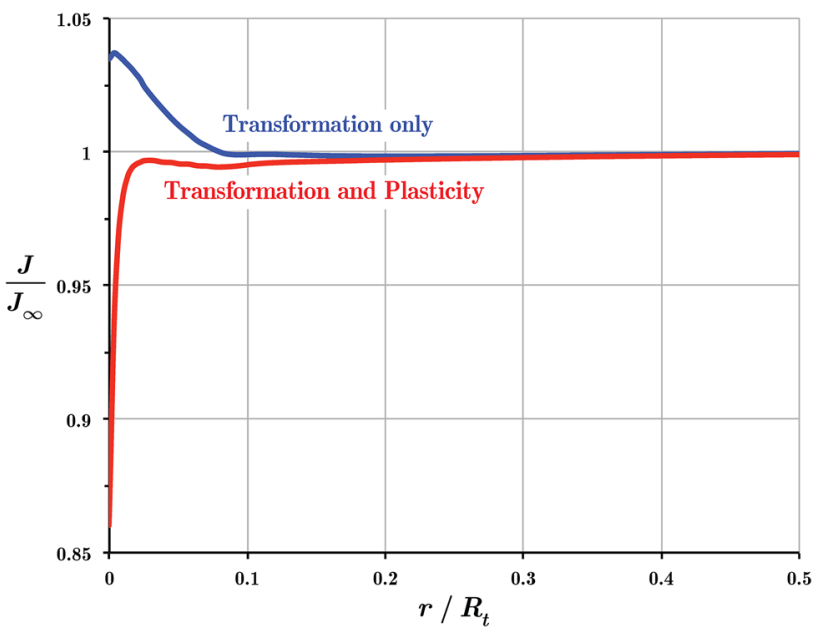

Fig. 5 The $J$-integral calculated along circular paths at different radii around the crack tip for the material with only pseudoelastic transformation, and with both transformation and plasticity

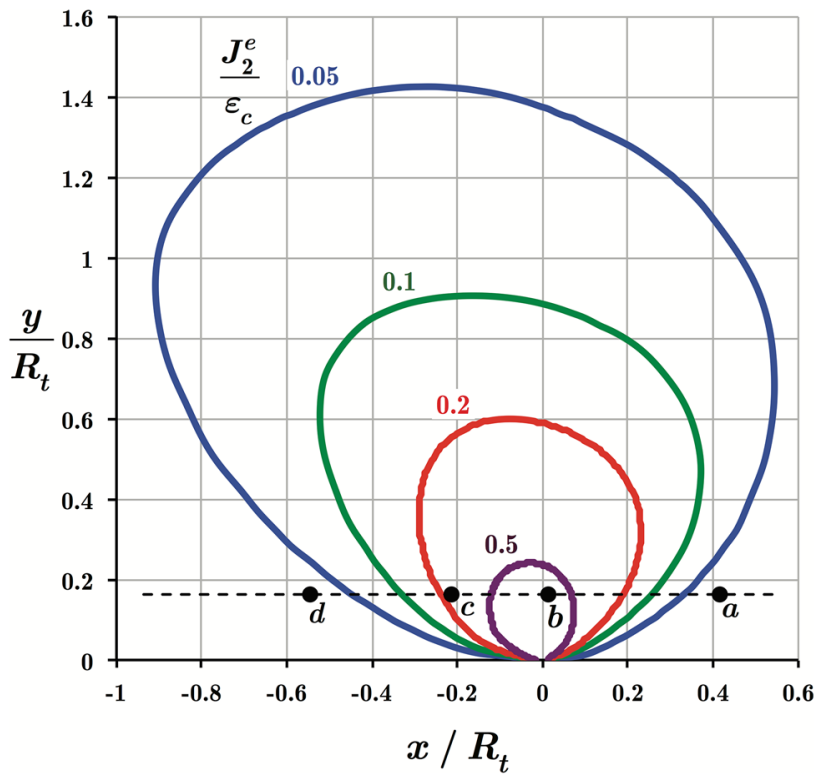

Fig. 6 Contours of equivalent transformation strain around the tip of a steadily propagating mode I crack (the material has no plastic deformation). The dashed line with indicated points is the path that a given material point at $y / R_{t}=0.16$ will experience as the crack passes through the material. The stress and remanent strain histories for this material point are shown in Figs. 7 and 8

contours of equivalent transformation strain $J_{2}^{e}$ normalized by $\varepsilon_{c}$ in the vicinity of a crack tip where the material has pseudoelastic transformation for the cases where there is and is not plastic deformation. The presence of plastic deformation has a nearly imperceptible effect on the transformation strain contours. Although these transformation strain contours are not perturbed significantly by the presence of plasticity, the effects of plasticity on the 

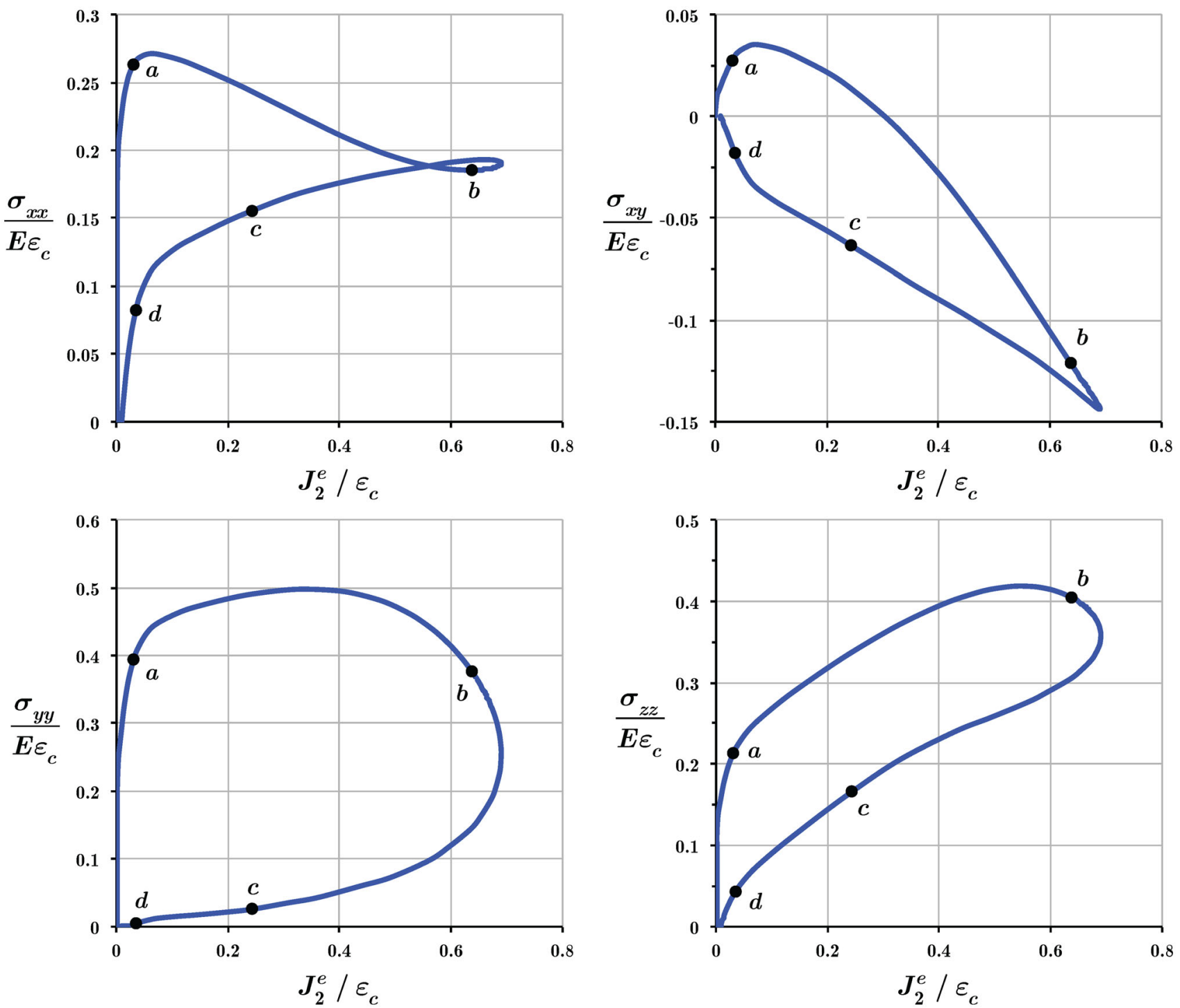

Fig. 7 The histories of stress components versus equivalent transformation strain of the points on the horizontal path marked as the dashed line in Fig. 6

$J$-integral near the crack tip are more apparent. The $J$ integral is given as,

$J=\int_{\Gamma}\left(W n_{1}-\sigma_{j i} n_{j} u_{i, 1}\right) d \Gamma$

where $W=\int \sigma_{i j} d \varepsilon_{i j}$ is the stress work density. Equation (36) is computed using a domain integral technique, Li et al. [33]. For these materials, proportional loading is not guaranteed, and hence the $J$-integral is not path-independent. However, the domain integral technique yields the averaged value of $J$ across the domain annulus and provides an accurate approximation of $J$ at the center of the annulus, Carka and Landis [34, 35].

Figure 5 plots the $J$-integral calculated along circular paths at different radii around the crack tip for the material model with only pseudoelastic transformation, and with both transformation and plasticity. These behaviors of the $J$-integral mimic those seen in ferroelastic materials, Carka and Landis [35], and in elastic-plastic materials, Carka and Landis [34]. Without plasticity, there is an elevation of the $J$-integral near the crack tip that we postulate is due to the strain saturation behavior that is observed in both ferroelastic and pseudoelastic materials. In contrast, when plastic deformation is included in the model, the $J$-integral drops off within the plastic zone to approximately $86 \%$ of its far-field value, which has also been observed in simple elastic-plastic materials.

\section{Steadily Propagating Cracks}

In contrast to material points near stationary cracks which experience nearly proportional load paths as the applied $K$ field is increased, material points around propagating cracks are subjected to highly non-proportional load paths. 

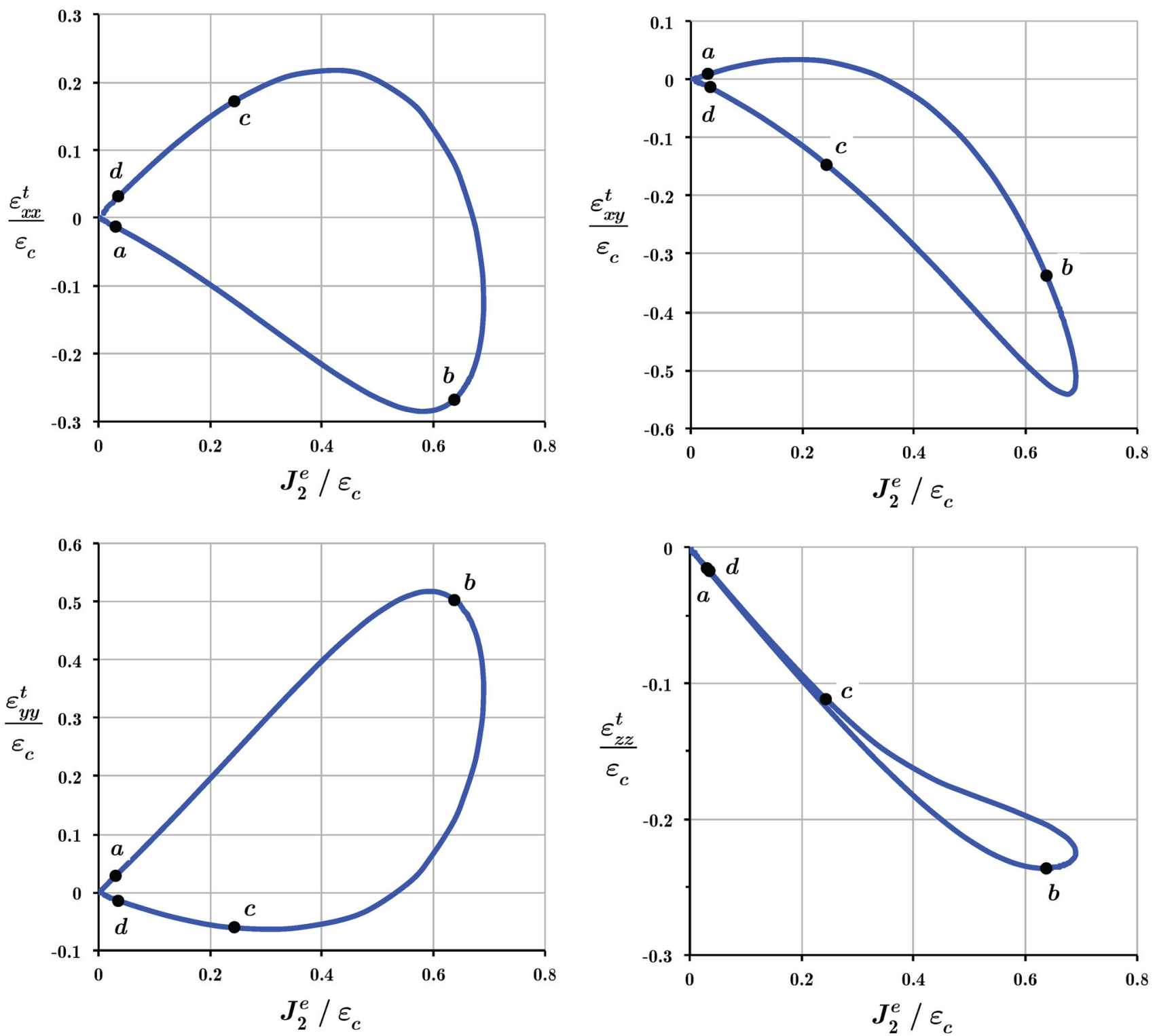

Fig. 8 The histories of transformation strain components versus equivalent transformation strain of the points on the horizontal path marked as the dashed line in Fig. 6

As for the stationary crack calculations, these calculations are for a semi-infinite crack propagating through a pseudoelastic material subjected to a far-field applied $K_{I}$. Here, the steady-state crack growth formulation pioneered by Dean and Hutchinson [36] is implemented. Additionally, the $J$-integral of (36) becomes the $I$-integral for steady state, Hutchinson [37]. For steady state, the $I$-integral is always path-independent and for ferroelastic and ferroelectric materials can be used to determine the ratio of the crack tip energy release rate to the far-field applied energy release rate. This procedure has been used by Landis [18] for ferroelastic materials, Wang and Landis [38, 39] for ferroelectric materials, and Baxevanis et al. [40, 41] for pseudoelastic materials. For the material properties listed in Table 1 with plastic deformation turned off, the computed ratio of the far-field steady-state energy release rate to the crack tip energy release is,

$\frac{G_{s S}}{G_{\text {tip }}}=2.34$

which means that if the material abides by a mode I propagation criterion such that the crack grows when the crack tip energy release rate achieves a critical values, then the results in Fig. 5 along with (37) imply that this material will exhibit $\mathrm{R}$-curve behavior with the plateau of the R-curve approximate 2.3 times higher than the growth initiation value. 
Figure 6 illustrates several contours of equivalent transformation strain $h_{1}^{p}$ normalized by $\sigma_{y} /\left(E \varepsilon_{c}\right)$ in the vicinity around the tip of the steadily propagating crack. Note that the outermost contour is $30-40 \%$ larger than the analogous contour for the stationary crack, but that the inner contours representing the more intense deformation are similarly sized. Also notice that there is no wake of deformed material behind the active transformation zone, in contrast to the case for ferroelastic, ferroelectric, and elastic-plastic materials. This is a manifestation of the pseudoelastic behavior, wherein the transformation strain returns to zero along with the stress. We also note that the present constitutive model allows this to occur for highly non-proportional loading paths as is illustrated in Figs. 7 and 8.

Figures 7 and 8 show the histories of stress and transformation strain components versus the equivalent transformation strain of the material point that traverses the horizontal path marked as a dashed line in Fig. 7. Any point on this line ahead of the crack will undergo the histories in the order of $a-b-c-d$ as the crack propagates along the $x$-axis. From the spatial perspective, these subfigures also present the distribution of stresses vs. equivalent transformation strain along the horizontal path.

Note that the stress and transformation strain history experienced by this material point is highly non-proportional. This means that the material point undergoes both an initial transformation from austenite to martensite as the point enters the transformation zone, but then also experiences a reorientation of the martensite as the point progresses through the transformation zone.

\section{Discussion}

A new constitutive model for isothermal pseudoelastic behavior was presented. The framework of the theory follows the one that was previously developed for ferroelastic and ferroelectric switching behavior. The basis is a flow-theory, plasticity-like description with a transformation surface, associated flow rule, and kinematic hardening with back stresses derived from a transformation potential. In order to allow for plastic deformations at high stresses, a standard $J_{2}$ based yield surface with isotropic hardening is also included. In contrast to many prior theories on pseudoelastic behavior, this theory does not identify the martensite volume fraction as one of the internal variables. Instead, a single transformation surface is used to capture both the effects of initial transformation from austenite to martensite and the reorientation of existing martensite. However, we note that the partitioning of these processes is not explicitly made within this phenomenological framework. Consequently, the ultimate test of the theory is on how well it is able to reproduce experimental observations.
This paper does not provide for such comparisons to experiments, but our prior work on modeling the buckling and recovery of NiTi tubes, Jiang et al. [23, 24], has demonstrated that the application of this model to that problem yields good agreement between calculations and observations when tension-compression asymmetry in stress and strain, and plasticity are incorporated within the calculations. Furthermore, the stress histories in the buckled tube, like the steady-state crack growth problem, are highly non-proportional going from compression in the axial direction prior to buckling to tension in the hoop direction after the formation of buckle lobes. In this work, we demonstrate the capabilities of the theory for some additional problems for the fields around crack tips. The stationary cracks solutions illustrate the effects that plasticity can have in regions where stresses and strains are high, and the steady crack growth calculations demonstrate the ability of the model to simulate highly non-proportional loading states.

Acknowledgments The authors acknowledge with thanks the financial support received for this work from the National Science Foundation under Grant No. CMMI-12000465.

\section{References}

1. Shaw JA, Kyriakides S (1995) Thermomechanical aspects of NiTi. J Mech Phys Solids 43:1243-1281

2. Bhattacharya K (2003) Microstructure of martensite: why it forms and how it gives rise to the shape-memory effect. Oxford University Press, Oxford

3. Gall K, Sehitoglu H, Chumlyakov YI, Kireeva IV (1999) Tension-compression asymmetry of the stress-strain responses in aged single crystal and polycrystalline NiTi. Acta Mater. 47:1203-1217

4. Bechle NJ, Kyriakides S (2014) Localization in NiTi tubes under bending. Int J Solids Struct 51:967-980

5. Reedlunn B, Churchill CB, Nelson EE, Shaw JA, Daly SH (2014) Tension, compression, and bending of superelastic shape memory alloy tubes. J Mech Phys Solids 63:506-537

6. Lagoudas DC, Entchev PB, Popov P, Patoor E, Brinson LC, Gao X (2006) Shape memory alloys. Part II: Modeling of polycrystals. Mech Mater 38:430-462

7. Patoor E, Lagoudas DC, Entchev PB, Brinson LC, Gao X (2006) Shape memory alloys. Part I: General properties and modeling of single crystals. Mech Mater 38:391-429

8. Boyd JG, Lagoudas DC (1996) A thermodynamical constitutive model for shape memory materials. Part I. The monolithic shape memory alloy. Int J Plast 12:805-842

9. Qidwai MA, Lagoudas DC (2000) On thermomechanics and transformation surfaces of polycrystalline NiTi shape memory alloy material. Int J Plast 16:1309-1343

10. Auricchio F, Petrini L (2004) A three-dimensional model describing stress-temperature induced solid phase transformations: solution algorithm and boundary value problems. Int J Numer Methods Eng 61:807-836

11. Arghavani J, Auricchio F, Naghdabadi R, Reali A, Sohrabpour S (2010) A 3-D phenomenological model for shape memory alloys under multiaxial loadings. Int J Plast 26:976-991

12. Arghavani J, Auricchio F, Naghdabadi R, Reali A, Sohrabpour S (2010) A 3D finite strain phenomenological model for shape memory alloys considering martensite reorientation. Contin Mech Thermodyn 22:345-362 
13. Panico M, Brinson LC (2007) A three-dimensional phenomenological model for martensite reorientation in shape memory alloys. J Mech Phys Solids 55:2491-2511

14. Brocca M, Brinson LC, Bazant ZP (2002) Three-dimensional constitutive model for shape memory alloys based on microplane model. J Mech Phys Solids 50:1051-1077

15. Karamooz Ravari MR, Kadkhodaei M, Ghaei A (2015) A microplane constitutive model for shape memory alloys considering tension/compression asymmetry. Smart Mater Struct 24:075016

16. Landis CM (2002) Fully coupled, multi-axial, symmetric constitutive laws for polycrystalline ferroelectric ceramics. J Mech Phys Solids 50:127-152

17. Landis CM (2003) On the strain saturation conditions for polycrystalline ferroelastic materials. ASME J Appl Mech 70:470-478

18. Landis CM (2003) On the fracture toughness of ferroelastic materials. J Mech Phys Solids 51:1347-1369

19. Sehitoglu H, Karaman I, Anderson R, Zhang X, Gall K, Maier HJ, Chumlyakov Y (2000) Compressive response of NiTi single crystals. Acta Mater. 48:3311-3326

20. Ezaz T, Wang J, Sehitoglu H, Maier HJ (2013) Plastic deformation of NiTi shape memory alloys. Acta Mater 61:67-68

21. Hartl DJ, Lagoudas DC (2009) Constitutive modeling and structural analysis considering simultaneous phase transformation and plastic yield in shape memory alloys. Smart Mater Struct 18:104017

22. Lagoudas DC, Entchev PB (2004) Modeling of transformationinduced plasticity and its effect on the behavior of porous shape memory alloys. Part I: constitutive model for fully dense SMAs. Mech Mater 36:865-892

23. Jiang D, Bechle N, Landis CM, Kyriakides S (2016) Buckling and recovery of NiTi tubes under axial compression. Int J Solids Struct 80:52-63

24. Jiang D, Landis CM, Kyriakides S (2016) Effects of tension/compression asymmetry on the buckling and recovery of NiTi tubes under axial compression. Int J Solids Struct. doi:10. 1016/j.ijsolstr.2016.07.003

25. Sedlak P, Frost M, Benesova B, Zineb TB, Sitnner P (2012) Thermomechanical model for NiTi-based shape memory alloys including R-phase and material anisotropy under multi-axial loadings. Int J Plast 39:132-151

26. Lexcellent C, Vivet A, Bouvet C, Calloch S, Blanc P (2002) Experimental and numerical determinations of the initial surface of phase transformation under biaxial loading in some polycrystalline shape-memory alloys. J Mech Phys Solids 50:2717-2735

27. Christ D, Reese S (2009) A finite element model for shape memory alloys considering thermomechanical couplings at large strains. Int J Solids Struct 46:3694-3709

28. Cazacu O, Plunkett B, Barlat F (2006) Orthotropic yield criterion for hexagonal closed packed metals. Int J Plast 22:1171-1194

29. Zaki W (2010) An approach to modeling tensile-compressive asymmetry for martensitic shape memory alloys. Smart Mater Struct 19:025009

30. Aleong D, Dumont C, Chirani SA, Patoor E, McDowell DL (2002) Transformation surfaces of a textured pseudoelastic polycrystalline $\mathrm{Cu}-\mathrm{Zn}-\mathrm{Al}$ shape memory alloy. J Intell Mater Syst 13:783-793

31. Baxevanis T, Chemisky Y, Lagoudas DC (2012) Finite element analysis of the plane strain crack-tip mechanical fields in pseudoelastic shape memory alloys. Smart Mater Struct 21(9):094012

32. Carka D, Mear ME, Landis CM (2011) The dirichlet-to-neumann map for two-dimensional crack problems. Comput Methods Appl Mech Eng 200:1263-1271

33. Li FZ, Shih CF, Needleman A (1985) A comparison of methods for calculating energy release rates. Eng Fract Mech 21:405-421

34. Carka D, Landis CM (2011) On the Path-dependence of the $J$ integral in an elastic-plastic material. J Appl Mech 78:011006

35. Carka D, Landis CM (2011) The analysis of crack tip fields in ferroelastic materials. Smart Mater Struct 20:094005

36. Dean RH, Hutchinson JW (1980) Quasi-static steady crack growth in small scale yielding. Fracture Mechanics, ASTM-STP 700:383-405

37. Hutchinson JW (1974) On steady quasi-static crack growth. Harvard University Report, Division of Applied Sciences, DEAP S-8

38. Wang J, Landis CM (2006) Effects of In-plane electric fields on the toughening behavior of ferroelectric ceramics. J Mech Mater Struct 1:1075-1095

39. Wang J, Landis CM (2006) Domain switch toughening in polycrystalline ferroelectrics. J Mater Res 21:13-20

40. Baxevanis T, Landis CM, Lagoudas DC (2014) On the fracture toughness of pseudoelastic shape memory alloys. J Appl Mech 81:041005

41. Baxevanis T, Landis CM, Lagoudas DC (2014) On the effect of latent heat on the fracture toughness of pseudoelastic shape memory alloys. J Appl Mech 81:101006 